\title{
Functional assessment in athletes with and without knee injury of a rugby team
}

\begin{abstract}
Rugby is one of the most practiced sports in the world and under development in Brazil, being one of the most expanding. It is a collision sport and extremely competitive, exposing players to a high number of injuries, especially in the knee joint. The aim of this study was to evaluate the prevalence of injuries in the anterior cruciate ligament or posterior cruciate ligament of the knee, the type of treatment of choice (conservative or surgical), and functional performance of amateur rugby athletes from a municipality in the Vale do Rio region. of the bells. Methodologically, it was characterized by an observational, cross-sectional study with a quantitative approach. Data collection was performed with amateur rugby players from a municipality in the Rio dos Sinos Valley region. To perform this research, two questionnaires were used as instruments, one for characterization of the sample profile and the other for subjective knee evaluation through the IKDC-2000, in addition to functional tests - Hop Tests. Sixteen amateur rugby athletes with a mean age of $23.75 \pm 4.43$ years participated in the study. The results show that most of the athletes $(81.3 \%)$ were male, that of the 16 athletes who participated, only $3(18.8 \%)$ presented knee ligament injury (ACL or PCL), and that of all athletes who presented knee injury, $100 \%$ were male. Among those with injuries, $12.5 \%$ underwent conservative treatment and $6.3 \%$ underwent surgical treatment. Regarding functional performance, all athletes presented a high level of lower limb symmetry index in functional tests, regardless of presenting ligament injuries or not, with no statistical difference $(p<0.05)$. Regarding the IKDC-2000 score, they had a regular performance, with an average of $75.46 \pm 15.31 \%$, demonstrating that the athletes present a regular performance, but it does not hinder its functionality in relation to the activities performed. It was possible to identify, from the analyzed data, that before the sample there were few athletes with previous knee ligament injury, besides all presenting, being classified as partial ruptures. This may be due to the fact that athletes with more significant injuries end up withdrawing from sports or are functionally well. However, there is a need for follow-up to prevent future injuries, considering that of the sixteen athletes, only seven perform injury prevention work.
\end{abstract}

Keywords: athletes, injuries, olympic sports, rugby players, ligament reconstruction, thraumatological injuries
Volume 5 Issue I - 2020

\author{
Nicolle Lahm Barbosa,' Leonardo Fratti \\ Neves $^{2}$ \\ 'Student, Department of Physical Therapy, Feevale University, \\ Brazil \\ ${ }^{2}$ Professor, Department of Physical Therapy, Feevale University, \\ Brazil
}

Correspondence: Leonardo Fratti Neves, Professor, Department of Physical Therapy, Feevale University, Brazil, Email leo_onevs@hotmail.com

Received: January 16, 2020 | Published: January 29, 2020
Abbreviations: IRB, international rugby board; ACL, anterior cruciate ligament; PCL, posterior cruciate ligament; IKDC, international knee documentation committee

\section{Introduction}

Rugby is one of the most practiced sports in the world and under development in Brazil, being one of the most expanding sports in the country, but there are still few Brazilian competition teams. ${ }^{1,2}$ In 2016 , its growth in the country was visible due to its insertion in the Olympic sports, participating for the first time in the Rio Olympics, having a high increase of clubs, federated athletes and social projects involving this one. sport mode. ${ }^{3,4}$ The Brazilian women's team has stood out worldwide, including more than the men's team. They are in the tenth position of the ranking of the International Rugby Board (IRB), ${ }^{5}$ bringing to Brazil for its performance in 2014, one of the stages of the world circuit of the sport, being a great achievement for the growth of rugby in the country. ${ }^{3}$

It is a collision sport and extremely competitive, which originated in 1823 , in the city of Rugby being the second most practiced sport in the world, behind only football. It is played on a football-like field where 15 players participate in each team, in two halves of 30 to 40 minutes each, being separated by an interval of 10 minutes. The physiological demands of rugby are complex, requiring players to have maximum agility, speed, strength, muscle power and aerobics. Being a sport of frequent body contact, they expose the players to a high number of injuries, especially in the knee joint. According to the literature, injury rates may be higher in amateur rugby players, considering differences in skills and abilities, ground conditions, refereeing patterns and attitudes of aggression and violence., ${ }^{2,6,7}$

Static stability of the knee joint depends on four major ligaments, which provide primary restriction to abnormal knee movement. These include the anterior cruciate ligament (ACL) and posterior cruciate ligament (PCL). ${ }^{8}$ Ligament injuries occur most frequently in individuals between 20 and 40 years of age, as a result of sports injuries. ${ }^{9}$ The knee is one of the most injured joints in the body. There are several types of injuries found clinically in this joint, including knee ligament injuries. ${ }^{8}$ Although ACL injuries have been reported in almost every athletic activity, it is in sports that include running and jumping that the highest numbers are found. In PCL injuries, in the first circumstance, the injury is seen in contact and collision sports. In these cases, the most significant aspect is the static and dynamic evaluation. These include static tests such as the anterior and posterior drawers, the Lachman test, among others. ${ }^{10}$ In the dynamic evaluation, we can mention the Hop Tests, which evaluate lower limb strength and confidence, aiming to assess the return to play capacity of the 
injured knee when compared with the uninjured contralateral limb, especially after ligament reconstruction. ${ }^{11}$

ACL and PCL injury rates for military and professional athletes are considerably higher, and rates for amateur athletes are moderately higher than national population incidence rates. Based on this, it is important to evaluate the incidence of rugby injuries and functional status of these athletes. ${ }^{12}$

\section{Methods}

The study was submitted and approved by the Feevale University Ethics Committee, under opinion 2,858,239 and CAAE: 91846418.1.0000.5348. Sixteen male and female amateur rugby athletes from a team were analyzed, with a mean age of $23,75 \pm 4,43$ years. As inclusion criterion for this study, amateur rugby athletes who accepted to participate in the study and signed an informed consent form (ICF), aged between 18 and 45 years, who trained at least twice a week and who participated of championships by the club were selected. Athletes who refused to participate in the research or did not sign the ICF, who were not found during the team visits to perform the questionnaires and functional tests, and athletes who presented some impossibility to perform the tests, functional tests or presented recent thraumatological injuries were excluded.

The athletes answered a sample profile questionnaire prepared by the researchers, which addresses sociodemographic information, injury prevalence and athlete training profile. In addition, a subjective knee assessment form was applied (International Knee Documentation Committee - IKDC version 2000) to assess functionality, quality and pain in developing daily living activities (DLAs) and sports activities for athletes who have presented knee ligament rupture injuries. Functional lower limb tests, such as single hop test for distance, crossover hop test for distance, triple hop test for distance, and 6-meter hop test for time were also performed to evaluate the power and stability of the lower limbs.

For the performance of the functional tests, a warm-up of 5 minutes was initially performed with each athlete, where they were instructed to run on the field in their own pace. Warming up directly interferes by reducing the risk of athletes experiencing muscle and joint pain during the tests. First, a single hop test for distance was performed, which consists of a single forward jump, leaving behind the previously demarcated line, and using the upper limbs for thrust, landing with the same limb and remaining stabilized for 2 seconds. Then the triple hop test for distance was performed, where it also left the previously demarcated line, using the upper limbs to propel it in the jump. The athlete should perform three consecutive hops, with the same leg, landing and remaining stabilized for 2 seconds on the last one. The next test performed by the athlete was the crossover hop test for distance, where the athlete was asked to stay behind the demarcated line, the limb to be tested and perform 3 subsequent jumps, crossing the fixed line in front of him with the foot and stabilizing on the limb being tested, remaining stable for 2 seconds. Finally, the 6-meter hop test for time was performed where the researcher asked the athlete to leave behind the previously demarcated line and jump on a single limb within 6 meters as quickly as possible, using the upper limbs to propel him. All tests were performed 3 times for each limb, with one attempt for familiarization before. The best distance jump was used for the single hop test for distance test, triple hop test for distance test and crossover hop test for distance test. For the 6 meters hop test for time, the fastest time of the three attempts was used as a result. Tests were invalidated when the athlete could not stabilize on landing, touched the ground with the contralateral foot or upper limbs or touched the starting line.

A database was created for statistical analysis. Data were analyzed in the Statistical Package for Social Science for Windows (SPSS) 21.0. Descriptive variables were presented as mean and standard deviation, as well as absolute numbers and percentages. Shapiro Wilk test was used to assess data normalization $(\mathrm{p}<0,05)$. Appropriate statistical tests were applied for means comparisons, such as the t-student or ANOVA test, as well as correlations between numerical variables and the chi-square test for categorical variables $(p<0,05)$.

\section{Results}

The average age of the athletes was $23,75 \pm 4,91$ years, with the lowest age being 18 years and the largest 31 years. The average weight presented by the participants was $89,26 \pm 22.69 \mathrm{Kg}$ ( 50 to $130 \mathrm{Kg}$ ), and the average height was $1,78 \pm 0,08 \mathrm{~m}(1,63$ to $1,92 \mathrm{~m})$, (Table 1$)$. When evaluating the body mass index (BMI), an average of $27,62 \pm 5.43$ $\mathrm{Kg} / \mathrm{m}^{2}$ was present $\left(18,14\right.$ to $\left.37,18 \mathrm{Kg} / \mathrm{m}^{2}\right)$. The sample consisted of 16 amateur rugby players, in which $3(18.8 \%)$ were female and 13 $(81.3 \%)$ male. The athletes' practice time was $59,81 \pm 36,1$ months $(1$ to 120 months). The average training time per week was $3,12 \pm 1,43$ hours ( 2 to 8 hours), as shown in Table 1. Regarding the dominant side of the athletes $13(81.3 \%)$ were right-handed and $3(18.8 \%)$ were left-handed.

Table I Biodemographic profile and training characteristics of amateur rugby athletes $(n=16)$

\begin{tabular}{ll}
\hline & Mean \pm SD \\
\hline Age (years) & $23,75 \pm 4,43$ \\
Weight $(\mathrm{Kg})$ & $89,26 \pm 22,69$ \\
Height $(\mathrm{m})$ & $1,78 \pm 0,08$ \\
BMl $\left(\mathrm{Kg} / \mathrm{m}^{2}\right)$ & $27,62 \pm 5,43$ \\
Time of practice (months) & $59,8 \mathrm{I} \pm 36, \mathrm{I}$ \\
Training time (hours per week) & $3,12 \pm \mathrm{I}, 43$ \\
\hline
\end{tabular}

\section{$\mathrm{SD}$, side deviation}

The athletes were divided by different positions considered as "advanced", "backward" and without defined position. Within the "advanced" group are the subgroups characterized by the positions Hooker, Pillar, Second Line, Wing and Eighth. Those considered "backwards" are presented as Medium Scrum, Tip, Aperture and Full Back. Regarding the type of knee ligament injury, 18,8\%(3) presented ligament injury. They were $66,7 \%(2)$ in the anterior cruciate ligament (ACL), and 33,3\%(1) in the posterior cruciate ligament (PCL). The athletes who presented previous knee ligament injury were $100 \%$ male. Regarding the movement performed at the time of the injury, $12,5 \%(2)$ of the athletes were injured in the tackle, and 6,3\%(1) within the ruck movement.

When asked if they had any kind of limitation after injuries $100 \%(3)$ of athletes who had injuries answered that they had no limitations. Regarding the treatment performed after injury, of the 16 amateur rugby athletes evaluated only $18,8 \%$ (3) presented injuries, and $12,5 \%(2)$ of the athletes underwent conservative treatment, and only $6,3 \%$ (1) athlete underwent surgical treatment. Regarding 
preventive treatment, $56,3 \%(9)$ do not perform any preventive exercises and $43,8 \%(7)$ perform preventive exercises. Of the athletes who presented ligament injury in this study $(18,8 \%)$, only $6,3 \%(1)$ reported doing preventive exercises.

Regarding the results of the Hop Tests, the athletes performed well, and the average symmetry index (SI) was $98,31 \pm 8,86 \%$ in the Single Hop Test, $99,85 \pm 5,02 \%$ in the Triple Hop Test, 103,31 $\pm 11,63 \%$ in the Cross Over Hop Test and 100,94 $\pm 11,17 \%$ in the Timed Hop Test. To have a good and positive result, with low injuries risk, the athlete should present a score above $90 \%$ of SI. ${ }^{13,14}$
Regarding the absolute results of the Hop Tests, the athletes obtained an average of $2,10 \pm 0,55 \mathrm{~m}$ with the non-dominant member in the Single Hop Test and 2,14 $\pm 0,55 \mathrm{~m}$ with the dominant member. In the Triple Hop Test, with the non-dominant limb, the athletes presented an average of $5,50 \pm 0,80 \mathrm{~m}$, and with the dominant limb the average of $5,52 \pm 0,78 \mathrm{~m}$. In the Cross Over Hop Test, the mean for the non-dominant limb was $5,06 \pm 0,66 \mathrm{~m}$, and $4,98 \pm 0,92 \mathrm{~m}$ for the dominant limb. Finally, when performing the Timed Hop Test, they obtained an average of $1,84 \pm 0,28$ seconds in the non-dominant limb and 1,82 $\pm 0,23$ seconds in the dominant limb. All information regarding Hop Tests are shown in Table 2.

Table 2 Overall results of the single hop tests, triple hop, hop cross over and timed hop rugby amateur athletes $(n=16)$

\begin{tabular}{llll}
\hline Test & Mean \pm SD & Minimum & Maximum \\
\hline Single Hop Non-Dominant $(\mathrm{cm})$ & $2,10 \pm 0,55$ & 1,31 & 3,39 \\
Single Hop Dominant $(\mathrm{cm})$ & $2,14 \pm 0,55$ & 1,37 & 3,45 \\
Simetry Single Hop $(\%)$ & $98,31 \pm 8,86$ & 82,30 & 113,00 \\
Triple Hop Non-Dominant (cm) & $5,50 \pm 0,80$ & 3,77 & 6,75 \\
Triple Hop Dominant (cm) & $5,52 \pm 0,78$ & 3,75 & 6,95 \\
Simetry Triple Hop (\%) & $99,85 \pm 5,02$ & 91,11 & 107,00 \\
Cross Over Hop Test Non-Dominant (cm) & $5,06 \pm 0,66$ & 3,46 & 5,80 \\
Cross Over Hop Test Dominant (cm) & $4,98 \pm 0,92$ & 2,58 & 6,43 \\
Simetry Cross Over Hop Test $(\%)$ & $103,31 \pm 11,63$ & 86,87 & 134,11 \\
Timed Hop Test Non-Dominant (s) & $1,84 \pm 0,28$ & 1,52 & 2,43 \\
Timed Hop Test Dominant (s) & $1,82 \pm 0,23$ & 1,45 & 2,29 \\
Simetry Timed Hop Test (\%) & $100,94 \pm 11,17$ & 83,41 & 130,60 \\
\hline
\end{tabular}

\section{SD, side deviation}

Para Myers et al. ${ }^{15}$ atletas em idade universitária devem apresentar como resultados aos testes de apoio unipodal uma média de $1,92 \pm 20 \mathrm{~m}$ no Single Hop Test considerando o público masculino e, $1,49 \pm 17 \mathrm{~m}$ para o público feminino. No Triple Hop Test a média deve ser $6,32 \pm 72 \mathrm{~m}$ (masculino), e 4,70 $\pm 53 \mathrm{~m}$ (feminino). Já para o Cross Hop Test a média para o público masculino é de $5,70 \pm 75 \mathrm{~m}$, e para o público feminino 4,06 $\pm 54 \mathrm{~m}$. Como resultado do Timed Hop Test, deve-se obter uma média de $1,74 \pm 0,21$ segundos entre os atletas masculinos, e $2,13 \pm 0,20$ segundos entre as atletas femininas.
There was no statistical difference between the means of performance in the Hop Tests when comparing the athletes who had and did not have previous knee joint injuries. For comparison between athletes with and without knee injury, only the results of male athletes were used in the data analysis, considering that the results for female athletes have different scores, thus altering the result. The functional test data of amateur rugby athletes with and without ligament injury are presented in Table 3.

Table 3 Comparative results of single hop, triple hop, cross over hop and timed hop tests of amateur rugby athletes, with and without history of ACL or PCL injury $(n=16)$

\begin{tabular}{|c|c|c|c|}
\hline Test & Previous injury mean \pm SD & No previous injury mean \pm SD & $\mathbf{p}$ \\
\hline Single Hop Non-Dominant $(\mathrm{cm})$ & $2,13 \pm 0,46$ & $2,27 \pm 0,61$ & 0,718 \\
\hline Single Hop Dominant (cm) & $2,15 \pm 0,49$ & $2,32 \pm 0,53$ & 0,625 \\
\hline Simetry Single Hop (\%) & $99,54 \pm 3,73$ & $97,63 \pm 10,33$ & 0,766 \\
\hline Triple Hop Non-Dominant (cm) & $5,52 \pm 0,41$ & $5,77 \pm 0,67$ & 0,560 \\
\hline Triple Hop Dominant (cm) & $5,75 \pm 0,42$ & $5,73 \pm 0,54$ & 0,961 \\
\hline Simetry Triple Hop (\%) & $96,13 \pm 4,38$ & $100,58 \pm 5,0 \mid$ & 0,195 \\
\hline Cross Over Hop Test Non-Dominant (cm) & $5,09 \pm 0,62$ & $5,20 \pm 0,55$ & 0,77 I \\
\hline
\end{tabular}




\begin{tabular}{|c|c|c|c|}
\hline Test & Previous injury mean $\pm S D$ & No previous injury mean $\pm S D$ & $\mathbf{p}$ \\
\hline Cross Over Hop Test Dominant $(\mathrm{cm})$ & $4,88 \pm 0,76$ & $5,28 \pm 0,62$ & 0,366 \\
\hline Simetry Cross Over Hop Test (\%) & $104,68 \pm 4,26$ & $98,73 \pm 7,42$ & 0,221 \\
\hline Timed Hop Test Non-Dominant (s) & $|, 7| \pm 0, \mid I$ & $1,76 \pm 0,25$ & 0,744 \\
\hline Timed Hop Test Dominant (s) & $1,73 \pm 0,17$ & $1,75 \pm 0,18$ & 0,853 \\
\hline Simetry Timed Hop Test (\%) & $99,32 \pm 5,56$ & $101,18 \pm 13,75$ & 0,828 \\
\hline
\end{tabular}

$\mathrm{SD}$, side deviation

Regarding the Subjective Knee Evaluation Form (IKDC), only $18,8 \%$ (3) athletes answered the questionnaire, considering that only these $18,8 \%$ presented knee injuries (ACL or PCL). The athletes presented an average of $75,46 \pm 15,31 \%$ points out of the $100 \%$ possible, classified as a regular result. ${ }^{16}$ Being the lowest score of $58,6 \%$, and the highest of $88,5 \%$.

\section{Discussion}

In a study aiming to identify the personal values of rugby practitioners in the metropolitan area of Porto Alegre, including 104 athletes from rugby clubs in suoth Brazil, presented $83,7 \%$ of the athletes aged 18 to 32 years. Within this percentage $44,2 \%$ were between 18 and 24 years old, $28,8 \%$ between 25 and 28 years old, and only $6,7 \%$ of the participants were over 37 years old. ${ }^{17}$ The author also states that the greatest interest in the practice of this sport is shown by the younger audience. Most team sports strive for the homogeneity of athletes in their practice. However, in rugby there is a very wide range of individuals with different physical attributes and can play in the same team, since each position has very specific and distinct requirements. ${ }^{18}$ Precisely because it presents great variability of skills, it is characterized by a sport of great opportunity for individuals of all shapes, sizes, skills and ages. ${ }^{19}$

In a study with 46 male athletes, where they were divided according to their positions in "advanced" and "backward", the "advanced" group $(\mathrm{n}=24)$ presented a weight average of $96,02 \pm 13,44 \mathrm{~kg}$, average height of $1,80 \pm 0,06 \mathrm{~m}$, and a BMI with average of $29,54 \pm 4,17 \mathrm{~kg} / \mathrm{m}^{2}$. On the other hand, the group "backwards" $(\mathrm{n}=22)$ had an average weight of $86,97 \pm 15,82 \mathrm{Kg}$, an average height of $1,75 \pm 0.05 \mathrm{~m}$, and BMI presenting an average of $25,45 \pm 1,94 \mathrm{~kg} / \mathrm{m}^{2} .{ }^{18}$ According to the authors, it is common that there is a great variability of skills among athletes, considering that the "advanced" are considered the "conquerors of the ball", being involved in both static and dynamic ball dispute situations, having the need to develop and apply greater physical strength. Unlike the "backs" who are considered the "users of the ball", being more involved in running and unmarking situations. In conclusion, most "advanced" athletes have a higher body mass than the "back" athletes.

Soft tissue injuries account for more than $50 \%$ of all rugbyassociated injuries, including musculotendinous injuries, ligament sprains and lacerations, bruises and bruises ${ }^{20}$. The authors also bring us that the lower limb is the most commonly injured anatomical region at all levels of experience, accounting for approximately $42 \%$ to $55 \%$ of all rugby injuries. As ACL injuries are the most common in the advanced group, causing a greater number of days away from the athletes. Knee injuries were the most common, with $20 \%$ of involvement, presenting as the most serious injury to ACL rupture, removing the athletes for an average of 235 days. PCL injuries are reported with a lower incidence and are conservatively treated. In this study of the $18,8 \%$ (3) of athletes who presented injury, $12,5 \%$ (2) play as "advanced", and 6,3\% (1) plays as "backward".

Another study which evaluated 62 rugby athletes, of which 51 athletes were male and 11 female, of the 62 athletes evaluated, 56,5\% $(n=35)$ athletes suffered injuries during the season, being $80 \%(n=28)$ men and $20 \%(n=7)$ women. ${ }^{4}$ The most frequent types of injuries among athletes were joint injury in $31,4 \%(\mathrm{n}=11)$ athletes, muscle injury in $28,6 \%(n=10)$ athletes, fracture in $25,7 \%(n=9)$. athletes and injury in $14,3 \%(n=5)$ athletes. Regarding location, $45,7 \%(n=16)$ of them suffered injury to the lower limb. According to the authors, most rugby injuries are musculoskeletal in nature and affect the lower limbs.

In another study, which evaluated 12 amateur rugby athletes, with an average age of 22,3 years, and 7 athletes presented injuries, being $51,1 \%$ of joint injuries, more frequently dislocations, tendon injuries, and ligaments, followed by muscle injuries $(21,5 \%)$, among the most common distension and contusion; fractures $(14,3 \%)$, and cutaneous lesions $(7,1 \%) .^{2}$ The traumas were mostly located in the lower limbs $(53,3 \%)$, most prevalent in the knee region.

Since rugby competition is growing international and national popularity, the sport has recently been associated with high levels of injury. ${ }^{20}$ Game speed and estimated injury incidence have doubled over the past 40 years. Most injuries occur during a tackle, with one player being tackled or facing another player. In addition, half of all injuries sustained during play occurred during a blind attack, outside the peripheral vision of the player being approached. The authors suggest that professional rugby players have a greater knowledge of the techniques and skills needed to prevent some of the most common injuries, while amateur athletes who are less qualified may be more prone to injury.

In one study, that evaluated 62 female $(19,4 \%)$ and male $(80,6 \%)$ rugby athletes, with a mean age of $19,5 \pm 6,2$ years. Of these 62 athletes, $56,5 \%$ suffered some injury during the season, and of these $31,1 \%$ underwent conservative treatment and $17,1 \%$ underwent surgical treatment. This is in line with this study, where most of the injured athletes, $12,5 \%(\mathrm{n}=2)$ underwent conservative treatment only, and $6,3 \%(n=1)$ underwent surgical treatment. ${ }^{4}$ Thus, other study in witch 12 athletes were evaluated with a mean age of $22,3 \pm 1,98$ years, 8 players attended the gym, 1 player performed specific muscle reinforcement to prevent a recurrent injury, and had the prevent injuries with warm-up and stretching before practice and games. ${ }^{2}$ Of the injured players, $71,4 \%$ did not perform injury prevention, which is in agreement with the data found in this study, where $56,3 \%(\mathrm{n}=9)$ of the athletes do not perform any preventive work. ${ }^{4}$ The authors also mention that athletes who have had a previous injury are more concerned with the habit of preventing injuries than those who have never suffered. 
Hop Tests can be predictive of knee joint injuries and are a useful tool for assessing the evolution of rehabilitation treatment ${ }^{21}$. It is the most used test in the evaluation of ACL reconstruction, especially for the athlete's easy understanding and execution. One research evaluated 80 athletes divided into two groups (ACL group and control group), with a mean age of $25,05 \pm 6,82$ years for the ACL group, and $27,7 \pm 8,16$ years for the control group, aiming to evaluate postural balance, muscle function of knee flexors and extensors and functional performance by Hop Test of athletes from different sports. ${ }^{21}$ They obtained as a result of the Single Hop Test in the preoperative phase an average of $1.23 \pm 0.38 \mathrm{~m}$ for the injured limb and $1.23 \pm 0.33 \mathrm{~m}$ for the non-injured limb, with an SI of $87 \%$. In the postoperative period, an average of $1,43 \pm 0,33 \mathrm{~m}$ for the operated limb and $1,39 \pm 0.27 \mathrm{~m}$ for the non-injured limb were presented, with an SI of $96 \%$. Regarding the control group, the average presented was $1,24 \pm 0,27 \mathrm{~m}$, with the SI of $102 \%$.

Deficits in lower limb neuromuscular control are a risk factor for secondary ACL injury, therefore, they are often used to assess readiness for return to sport, quadriceps strength, being associated the physical functioning of the limb after ACL reconstruction..$^{22}$ To assist in the athlete's return to sport, rehabilitation protocols have been developed, including Hop Tests. Being the most used as a return to sport. SI results tend to improve over time with almost all results above $90 \%$ within one year of primary ACL reconstruction.

We can see that in this study, regardless of having a knee ligament injury or not, all athletes had an SI above $90 \%$, which is the ideal value, brought by the literature as a good functional performance. There was no statistical difference between the results of the Hop Tests with the athletes who presented and those without ligament injuries (ACL and PCL). This is possibly because athletes who had more serious injuries ended up abandoning this sport or have a good functional performance before the tests.

Rugby is a contact game, where the player's body suffers impacts and collisions at high speed, which can result in different trauma. ${ }^{2}$ Therefore, it is considered a sport with a high injury rate compared to other sports. Therefore, this high number of physical collisions in which athletes are involved during the game justifies a high incidence of trauma.

$51,5 \%$ of the athletes analyzed in their study remain for an average

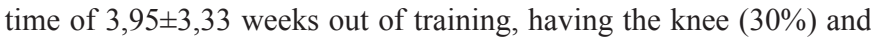
shoulder as the most affected structures $(25 \%){ }^{23}$ In 6 months of practice, more than half of the athletes remained for about 4 weeks out of sports due to injuries.

\section{Conclusion}

Rugby is a sport that involves intense physical contact and requires high level fitness. Muscle strength and power, speed and agility are important requirements for practicing athletes. It requires a variability of physiological responses from its players, as it is marked by repetitive high intensity runs and body contact. Therefore, it is a sport that has a high incidence of injuries, especially in amateur and semiprofessional leagues.

Regarding functional performance, all athletes presented a high level of SI, despite of their history of ligament injuries or not, with no statistical difference. In the IKDC, they had a regular performance, with an average score of $75,46 \%$.
It was possible to identify that there were few athletes with previous knee ligament injury. It can be inferred that athletes with more significant injuries or those with higher functional deficits end up moving away from sports, while those who remain with good functional performance continue to perform sports activity. However, there is a need for follow-up to prevent future injuries, considering that of the sixteen athletes, only seven perform injury prevention exercises. As a limitation, it can be considered that the analyzed group was restricted, with a relatively low number of participants.

\section{Funding}

None.

\section{Acknowledgements}

None.

\section{Conflicts of interest}

The authors declare no conflicts of interest.

\section{References}

1. Lopes André Luiz. Anthropometric and physiological profile of Brazilian rugby athletes. Brazilian Journal of Physical Education Sport. 2011;25(3):387-395.

2. Soares ASB, Silva MAAB, Liberalino EST. Prevalence of sports injuries in male athletes of the rugby team of the city of Caruaru/PE. 2016.

3. Moura GX. Historical retrospective of rugby expansion: from the world to maringá. International congress of pedagoia do esporte; 2015.

4. Toledo LED, Ejnisman B, Andreoli CV. Incidence, type and nature of injuries for athletes of são josé rugby in the 2014 Season. Revista Brasileira Medicina Esporte. 2015;21(3):215-219.

5. International rugby board. Guia de principiantes do rugby union. Disponível em. 2018.

6. Alves LM, Soares RP, Liebano RE. Incidence of injuries in the practice of amateur rugby in Brazil. Fisioterapia e Pesquisa. 2008;15(2):131135 .

7. Iwano TF. Incidence of injuries in rugby. course conclusion paper (Monograph) - physiotherapy course, Universidade Tuiuti do Paraná, Curitiba, PR, 2005:52.

8. Dutton M. Orthopedic physiotherapy. 2nd ed. São Paulo: Artmed SA; 2010:801-1006

9. Kisner C, Colby LA. Therapeutic exercises fundamentals and techniques. 6th ed. Barueri, SP: Manole; 2013;(21):764-848

10. Garrick JG, Webb DR, Sports injuries. 2nd ed. Sâo Paulo: Roca Ltda; 2001;(9):291-369.

11. Farias N, Demétryus C, Manso KP, et al. Comparison between HOP TEST and other tests used at discharge of patients with anterior cruciate ligament (ACL) injury. Rehabilitation and Health. 2017;19(38):11-16.

12. Logerstedt DS. Knee stability and moviment coorfination impairments: knee ligament sprain revision 2017. Journal of Orthopaedic Sports Physical Therapy. 2017;47(11):1-47.

13. Snyder J. Single- leg hop for distance. disponível. 2018.

14. Vasconcelos RA. Analysis of the correlation between peak torque, functional performance and ligament laxity in normal individuals and with reconstruction of the anterior cruciate ligament. Revista Brasileira Ortopedia. 2009;44(2):134-142. 
15. Myers B, Walter LJ, Peter R. Normative data for hop tests in high school and collegiate basketball and soccer players. The International Journal of Sports Physical Theraphy. 2014;9(5):596-603,

16. Silva Júnior, Ohashi BN, Almeida MO, et al. Functional result related to graft positioning in anterior cruciate ligament reconstruction. Revista Brasileira de Ortopedia. 2013;50(1):57-67.

17. Müller ÂF. Identification of the personal values of rugby players from clubs in the metropolitan region of porto alegre. course conclusion paper (Monograph) - Administration course, federal university of rio grande do sul, porto alegre, 2013. $65 \mathrm{p}$.

18. Ferreira AMC, Ribeiro CAF. Anthropometric and physiological profile of Portuguese rugby players - part I: comparison between athletes from different positional groups. Revista Bras Med Esporte. 2013;19(1):48512 .
19. World rugby. Leis do jogo rugby union. 2018.

20. Kaplan Kevin M et al. Rugby injuries: a reviewof of concepts and current literature. Bulletin of the NYU Hospital for Joint Diseases. 2008;2(66):86-93.

21. Felix ECR. Functional, isokinetic and posturographic analysis of athletes with anterior cruciate ligament injury before and after anatomical reconstruction with flexor tendon. Dissertation (Master in Experimental Pathophysiology) - Faculty of Medicine, University of São Paulo; 2017.

22. Abrams et al. Functional performance testing after anterior cruciate ligament reconstruction: a sistematic review. Orthop J Sport Med. 2014;2(1):1-10.

23. Kiefer T, Roberta AA, Correa AC, et al. Occurrence of absence from training due to injury in rugby athletes. Science \& Health Magazine. $2009 ; 24$. 